\title{
THE EXPERIMENTAL ASSESSMENT OF BUILD UP FACTOR AND ATTENUATION COEFFICIENT OF BRASS COMPENSATOR APPLIED IN INTENSITY-MODULATED RADIATION THERAPY (IMRT) FOR 6MV PHOTON BEAM
}

\author{
Elnaz $B^{1}$, Peyman $H^{2 *}$, Abbas $H^{3}$, Raheb $G^{4}$, Zaker $S^{5}$ \\ 1,2 Department of Medical Physics, Semnan University of Medical Sciences, Semnan, Iran \\ ${ }^{3}$ Department of Medical Physics, Kermanshah University of Medical Sciences, Kermanshah, Iran \\ ${ }^{4}$ Department of Epidemiology, Semnan University of Medical Sciences, Semnan, Iran \\ ${ }^{5}$ Department of Radiation Sciences, Lorestan University of Medical Sciences, Lorestan, Khorramabad, Iran
}

\author{
Correspondence: \\ Peyman Hejazi (PhD) \\ Semnan University of Medical Sciences, \\ Department of Medical Physics, \\ Semnan, Iran \\ Email: hejazi@semums.ac.ir
}

\begin{abstract}
Introduction: Recent compensators are commonly applied in IMRT. The precise properties of applied compensators such as thickness, attenuation coefficient and build up factor are intensively important for IMRT calculations.

Method: The brass compensator used for $6 \mathrm{MV}$ photon beam was studied to estimate the relative effect of thickness and field size on IMRT calculations. Various field size together with several compensator thicknesses were examined.

Result: The average reduction of effective attenuation coefficient (EAC), for the fields of $10 \times 10 \mathrm{~cm}^{2}$ to $20 \times 20$ $\mathrm{cm}^{2}$, was 9.94\%. By increasing the field size, EAC was decreased. The major reduction of EAC due to increasing field size was found to be $9.62 \%$. The build up factor was increased by $2 \%$ to $21.8 \%$ respect to field size and compensator thickness. Also, the build up factor was increased by adding up the thickness. The rate of changes ranged from $24 \%$ to $48 \%$.
\end{abstract}

Conclusion: The compensator thickness and field size are significantly important to calculate the effective attenuation coefficient and build up factor.

Keywords:Intensity Modulated Radiation Therapy, Compensators, Brass, Effective Attenuation Coefficient, Build up Factor

\section{Introduction:}

Radiotherapy is one of the effective methods in the treatment of cancer. It is used alone or in combination with surgery or chemotherapy. Half of the cancer patients use radiotherapy as a part of their treatment(1-3). The primary goal of radiotherapy is to deliver the highest dose to cancerous tissue and the lowest to normal organs(2, $4,5)$. As the tumor is not isolated, it is not possible to irradiate tumor cells alone. Also, success in removing the tumor depends on technical factors(6). In addition, for an appropriate treatment, accurate definition and description of tumor and treatment volume, high daily repeatability of patient positioning, and accurate dose delivery to the target volume with an appropriate dose of gradient to critical organs and healthy tissues are required. When some of these requirements are not met, a part of tumor may receive lower dose, and eliminate the chance of cancer cell proliferation would happen(7). In the recent methods of radiotherapy, intensity modulated radiotherapy (IMRT) technique is used, which needs very accurate calculations. In IMRT, the output of the beam must be accurately transferred to the depth of interest in tissue, while in different parts of the target volume and under risk organs in the beam path, various intensities of beam should be received. In other words, the uniform intensity of the output beam from the accelerator 
should be modulated in treatment volume $(8,9)$. Inverse planning method is used in IMRT to calculate the dose in the treatment volume. Target volume and critical organs are determined by CT scan images of tumors. Then the maximum, minimum, and average permissible doses are determined by TPS. TPS software propose several fields based on the optimization algorithm for which it is defined. In these fields, changes in the relative dose are indicated in each area, which show dose changes relative to the uniform open field. The process is called modulated intensity plan. To reach the dose levels, fields must be planned and implemented in accordance with the specification set via treatment planning software. TPS determines there are two ways to deliver radiation dose in IMRT: multileaf collimator and compensators(8-10). The advantages of using compensators are increasing the efficiency of patient treatment, and providing continuous dose. Other benefits of compensators are faster quality assurance program performance, easier dosimetry data management, less running time, less erosion of the accelerator, and lower requirement of shield in treatment room(11). Recently, tendency to use compensators for IMRT treatment has increased(12). The formula used to calculate the thickness of compensator is as follows(8):

$$
x=-\frac{1}{\mu_{e f f}} \ln \left(\frac{D}{D_{0}}\right)
$$

is the relative dose; $\mu_{\text {eff }}$ is the attenuation coefficient of the compensator, and $x$ is the thickness of the compensator(8). The thickness of the treatment planning software (TPS) can be sent to an automatic analysing system (such as, Parscientific, Model ACD-3, Odense, Denmark), and the compensator volume can be exploited(13). In the method of constructing compensators, using automatic analysing machines, the maximum reported error in the beam intensity as compared to the ideal state is $\pm 2.5 \%$, which is the half of acceptable error by ICRU(14).

The exact calculation for the optimal dose delivery with acceptable error level to the desired depth therapy is crucial to fabricate a compensator. therefore, the exact calculation of effective attenuation coefficient of the compensator is an important factor. Effective attenuation coefficient of the compensator is not only dependent on the material and the nominal energy of the accelerator, but also it changes by variations in radiation conditions. The other important factors influence on the attenuation coefficient of the compensator are the sizes of treatment field size and compensator thickness $(15,16)$. In several studies, $\mu_{\text {eff }}$ is calculated using various materials by Mont Carlo simulation (MC) (17-19) or experimental measurements(20) in various radiotherapy conditions. But the error rate in providing doses needs to be calculated due to the lack of the consideration of factors affecting attenuation coefficient of brass compensator. In the present research, after studying the changes in the effective attenuation coefficient based on the field size and compensator thickness, the error rate was calculated. The Buildup factor was also studied in this research. Considering to this fact that scattering particles are produced at the presence of compensator, therefore the build up factor which is used for counting the primary and scattered radiations can be applied for any definite geometry(21).

\section{Methods and Materials}

The applied brass alloys in this study was commercial brass contains:FCD (Cuzn39pb3) with 3\% lead, 61.5\% copper, and $35.5 \%$ zinc casted and constructed by cold rolling. Using dosimeter MapCHECK 2 model 1177, solid phantom, and SP34 (Solid Phantom 34), the effect of compensator thickness and field size on the effective attenuation coefficient and build up factor of the compensator was assessed by photon 6 MV Elekta SL 75/25 medical linear accelerator. For all irradiations carried out by $100 \mathrm{MU}$, the distance to phantom surface was set to $100 \mathrm{~cm}$. The brass compensator of various thicknesses was placed in the tray at the distance of $672 \mathrm{~mm}$ from the accelerator. Dose measurements in all conditions were carried out in solid water phantom at the depth of $5 \mathrm{~cm}$ (equivalent to tissue) by MapCHECK 2 dosimeter. To calculate the effective attenuation coefficient, Eq1. was applied. In this equation, $D$ is the measured dose in the field with compensator; $D_{0}$ is the measured dose in the field without compensator; $\mu_{\text {eff }}$ is the effective attenuation coefficient and $\mathrm{x}$ is compensator thickness was including $0.5,1,1.5,2,3,4,5$, and $6 \mathrm{~cm}$. The compensator was placed $672 \mathrm{~mm}$ in front of the head of gantry. In this section, the absorbed dose was measured with and without compensator at the depth of $5 \mathrm{~cm}$ in solid water phantom. Then, it was re-measured (D) for each thickness of the same depth. The field size for the thicknesses was ranged from $1 \times 1 \mathrm{~cm}^{2}$ to $20 \times 20 \mathrm{~cm}^{2}$. The effective attenuation coefficient of square fields for dimensions of $1,2,3,4,6,8,10,15$, and $20 \mathrm{~cm}$ and the thicknesses of $1,1.5,2,3,4,5$, and $6 \mathrm{~cm}$ was calculated by experimental measurements. For the photon beams, from $6 \mathrm{MV}$ to $18 \mathrm{MV}$ energy, the measurement error of MapCHECK dosimeter for dose values more than 8cGy was less than $1 \%(22)$. All the measurements were conducted for the photon beam of $6 \mathrm{MV}$ with the dose of $100 \mathrm{cGy}$.

\section{Build up Factor Calculations:}

The effective attenuation coefficient vs field size was depicted by Excel 2013. The proportional quadrative equation was then found. The linear attenuation coefficient was derived by extrapolation to field size of $0 \times 0$. The depth dose at this hypothetical field size results from primary radiation (scattered radiations will not change the depth dose at this field size). The build up factor of square fields for dimensions of $1,2,3,4,6,8,10,15$, and $20 \mathrm{~cm}$ and the thicknesses of $1,1.5,2,3,4,5$, and $6 \mathrm{~cm}$ was calculated by experimental measurements.

\section{Uncertainty in dose calculations:}

The following formula was used to calculate the error percentage: 
$\% \varepsilon=\left|\frac{e^{-\mu_{e f f}(x, F) x}-e^{-\mu_{e f f}\left(x=1 c m, F=10 \times 10 \mathrm{~cm}^{2}\right) x}}{e^{-\mu_{e f f}(x, F) x}}\right| \times 100$

Eq2.

$\mu_{\text {eff' }} \mathrm{x}, \mathrm{F}$ and $\varepsilon$ are effective attenuation coefficient, compensator thickness, field size and error level, respectively.

\section{Results:}

Figure 1 and Figure 2 show the changes in the effective attenuation coefficient versus compensator thickness and field size.

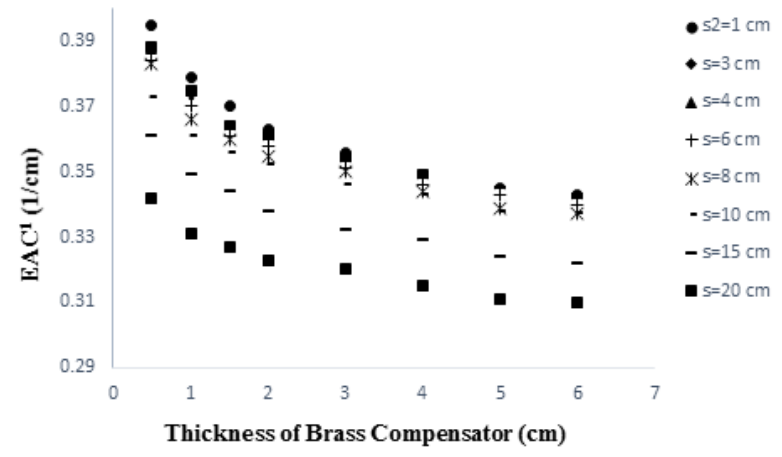

Figure 1. Variation of the $\mu_{\text {eff }}$ vs. compensator thickness for various field sizes.

${ }^{1}$ Effective Attenuation Coefficient

${ }^{2}$ Side of square field

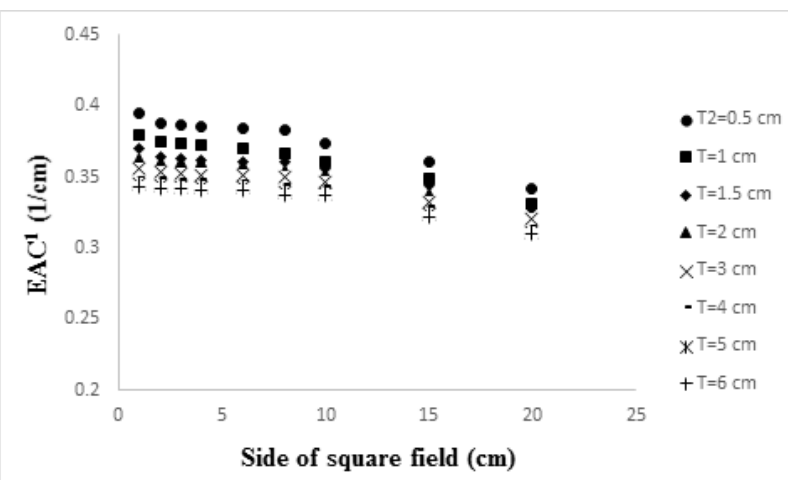

Figure 2. Variation of the $\mu_{\text {eff }}$ vs. the field size for various thicknesses of brass.

\footnotetext{
${ }^{1}$ Effective Attenuation Coefficient

${ }^{2}$ Thickness of compensator
}

The maximum acceptable error in radiotherapy is $5 \%$ that $3 \%$ of which is because of the error of measurements and dosimeter calculations, and the rest $2 \%$ refers to the treatment planning error(22). The error calculations, Table1, indicates that compensator thickness and field size potentially leads to more than $20 \%$ error in dose delivery of the treatment volume in the calculation of the effective attenuation coefficient.
Table 1. The error in dose delivery for thickness and field size

\begin{tabular}{|c|c|c|c|c|c|c|c|c|c|}
\hline & \multicolumn{8}{|c|}{ Thickness (cm) } \\
\hline & & $\begin{array}{l}0.5 \\
\mathrm{~cm}\end{array}$ & $\begin{array}{c}1 \\
\mathrm{~cm}\end{array}$ & $\begin{array}{l}1.5 \\
\mathrm{~cm}\end{array}$ & $\begin{array}{c}2 \\
\mathrm{~cm}\end{array}$ & $3 \mathrm{~cm}$ & $4 \mathrm{~cm}$ & $\begin{array}{c}5 \\
\mathrm{~cm}\end{array}$ & $\begin{array}{c}6 \\
\mathrm{~cm}\end{array}$ \\
\hline \multirow{9}{*}{ 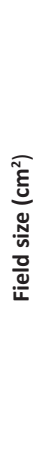 } & $1 \times 1 \mathrm{~cm}^{2}$ & 1.7 & 1.8 & 1.4 & 0.4 & 1.5 & 4.7 & 7.7 & 10.2 \\
\hline & $2 \times 2 \mathrm{~cm}^{2}$ & 1.4 & 1.4 & 0.5 & 0 & 2.1 & 4.7 & 8.1 & 10.8 \\
\hline & $3 \times 3 \mathrm{~cm}^{2}$ & 1.3 & 1.3 & 0.3 & 0.2 & 2.7 & 53.1 & 8.6 & 10.8 \\
\hline & $4 \times 4 \mathrm{~cm}^{2}$ & 1.2 & 1.1 & 0.2 & 0.2 & 3 & 5.1 & 8.6 & 11.3 \\
\hline & $6 \times 6 \mathrm{~cm}^{2}$ & 1.2 & 1 & 0 & 0.6 & 3 & 5.8 & 8.6 & 11.8 \\
\hline & $8 \times 8 \mathrm{~cm}^{2}$ & 1.1 & 0.5 & 0.2 & 1.2 & 3.2 & 6.6 & 10.4 & 13.4 \\
\hline & $10 \times 10 \mathrm{~cm}^{2}$ & 0.6 & 0 & 0.7 & 1.8 & 4.4 & 6.9 & 10.9 & 13.4 \\
\hline & $15 \times 15 \mathrm{~cm}^{2}$ & 0 & 1.2 & 2.5 & 4.5 & 8.3 & 12 & 16.9 & 20.9 \\
\hline & $20 \times 20 \mathrm{~cm}^{2}$ & 1 & 3 & 5 & 7.3 & 11.6 & 16.8 & 22.1 & 26.4 \\
\hline
\end{tabular}

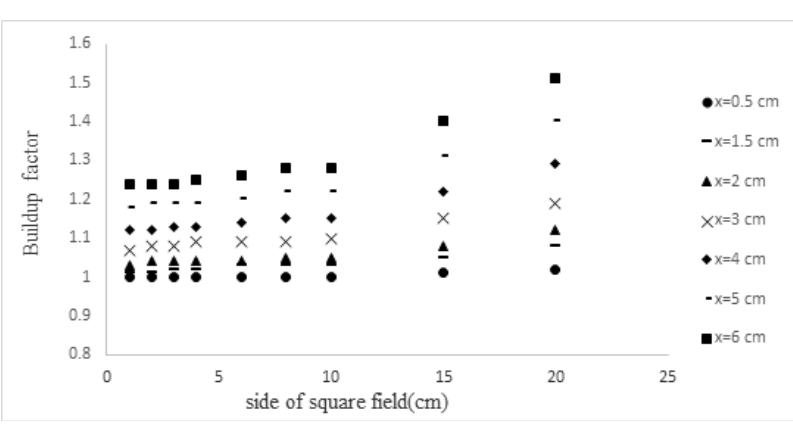

Figure 3. Variation of the buildup factor vs. the field size for various thicknesses of brass.

\section{Build up factor results:}

Figure 3 and Figure 4 show the changes of build up factor vs field size and thickness of brass compensator respectively.

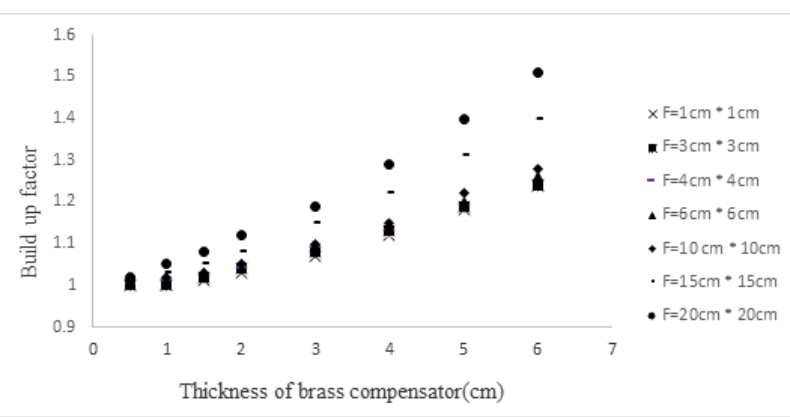

Figure 4. Variation of the buildup factor vs. compensator thickness for various field sizes.

\section{Discussion:}

The reduction in the effective attenuation coefficient by increasing thickness relates to the field size. As an average, $\mu$ eff decreased the effective attenuation coefficient by $11.57 \%$. For the fields of $10 \times 10 \mathrm{~cm}^{2}$ to $20 \times 20 \mathrm{~cm}^{2}$, the average reduction of the effective attenuation coefficient was $9.94 \%$ in Figure 1 . By increasing the field size, the 
effective attenuation coefficient was decreased. The major reduction of the effective attenuation coefficient due to increasing field size was found to be $9.62 \%$. This results is confirmed by a previous study carried out by $\mathrm{T}$. Bartrum and his team(16). They showed that there is a significant correlation between the effective coefficient of brass and field size for $6 \mathrm{MV}$ beam, so that the effective attenuation coefficient decreases with increasing field size. The results obtained by them show $2.5 \%$ disparity with the measurements of this study, which could be due to the difference in depth of measurement. By adding up the thickness from $0.5 \mathrm{~cm}$ to $2 \mathrm{~cm}$, the effective attenuation coefficient decreased by $12.18 \%$ averagely. By adding up the thickness from 2 to $6 \mathrm{~cm}$, the average decrease of the effective attenuation coefficient was obtained as 10.07 in Figure 2.

As it is illustrated in Figure 3, the build up factor is increased by $2 \%$ to $21.8 \%$ with field size and compensator thickness. Furthermore, the build up factor was increased by adding up the thickness (Figure 4). The rate of changes ranged from $24 \%$ to $48 \%$. One of the possible reasons for this increase is Compton scattering, considering to this fact that the probability of compton scattering increases with the number of electrons. It must be mentioned that the minimum and maximum values were obtained for the filed sizes of $1 \times 1$ and $20 \times 20$, respectively.

\section{Conclusion:}

In this study, the effect of changes in the thickness of brass compensator and field size on $\mu_{\text {eff }}$ and build up factor were assessed to be applied in IMRT. The results revealed that by increasing the thickness and field size, $\mu_{\text {eff }}$ was decreased. This study also demonstrated that the lack of consideration of compensator thickness and field size can lead to more than $20 \%$ error rate in dose delivery in the treatment volume. In other words, precise determination of compensator thickness and field size is significantly important for $\mu_{\text {eff }}$ calculation. The results of this study also showed that the build up factor increases by increasing field size and thickness of brass compensator.

\section{References:}

1. Souhami R, Tobias JS. Cancer and its management: John Wiley \& Sons. 2008.

2. Hopkins DN. Determination of the linear attenuation coefficients and buildup factors of MCP-96 alloy for use in tissue compensation and radiation protection: Ball State University. 2010.

3. Swinnen A. Quality assurance in radiotherapy: Development and validation of a mailed dosimetry procedure for external audits using a multipurpose phantom and in vivo dosimetry: Katholieke Universiteit Leuven. 2005.

4. Khadija M. A clinical comparison and analysis between conventional MLC based and solid compensator based IMRT treatment techniques. 2009.
5. Sasaki K, Obata Y. Dosimetrical characteristics of a cubic-block-piled compensator for intensitymodulated radiation therapy. Journal of Applied Clinical Medical Physics. 2007;8(1).

6. Halperin EC, Perez CA, Brady LW. Perez and Brady's principles and practice of radiation oncology: Lippincott Williams \& Wilkins. 2008.

7. Newhauser W. International Commission on Radiation Units and Measurements Report 78: Prescribing, Recording and Reporting Proton-beam Therapy. Radiation Protection Dosimetry. 2009.

8. Khan FM, Gibbons JP. Khan's the physics of radiation therapy: Lippincott Williams \& Wilkins. 2014.

9. Webb S. Intensity-modulated radiation therapy: CRC Press. 2001.

10. Webb S. The physical basis of IMRT and inverse planning. The British journal of radiology. 2014.

11. Bakal A, Laub W, Nusslin F. Compensators for IMRTAn Investigation in Quality Assorance. Zeitschrift fur Medizinische Physik. 2001;11(1):15-22.

12. Chang S. Compensator-intensity-modulated Radiotherapy-A traditional tool for modern application. US Oncological Disease. 2006:1-4.

13. Partridge $\mathrm{M}$, Donovan $\mathrm{E}$, Fenton $\mathrm{N}$, Reise S, Blane S. Clinical implementation of a computer controlled milling machine for compensating filter production. The British journal of radiology. 1999;72(863):1099103.

14. Meyer J, Mills J, Haas O, Parvin E, Burnham K. Some limitations in the practical delivery of intensity modulated radiation therapy. The British journal of radiology. 2000;73(872):854-63.

15. Dimitriadis D, Fallone B. Compensators for intensity-modulated beams. Medical Dosimetry. 2002;27(3):215-20.

16. Bartrum T, Bailey M, Nelson V, Grace M. Linear attenuation coefficients for compensator based IMRT. Australasian Physics \& Engineering Sciences in Medicine. 2007;30(4):281-7.

17. Du Plessis F, Willemse C. Monte Carlo calculation of effective attenuation coefficients for various compensator materials. Medical physics. 2003;30(9):2537-44.

18. Salehi Z, Ali NY, Yusoff A. X-ray spectra and quality parameters from Monte Carlo simulation and analytical filters. Applied Radiation and Isotopes. 2012;70(11):2586-9.

19. Jurkovic S, Zauhar G, Bistrovic M, Faj D, Kaliman Z, Radojcic DS. An alternative approach to compensators design for photon beams used in radiotherapy. Nuclear Instruments and Methods in Physics Research Section A: Accelerators, Spectrometers, Detectors and Associated Equipment. 2007;580(1):530-3.

20. Attix $\mathrm{FH}$. Introduction to radiological physics and radiation dosimetry: John Wiley \& Sons; 2008.

21. Li JG, Yan G, Liu C. Comparison of two commercial detector arrays for IMRT quality assurance. Journal of applied clinical medical physics. 2009;10(2). 
22. Jones D. ICRU report 50-prescribing, recording and reporting photon beam therapy. Medical physics. 1994;21(6):833-4. 